\title{
An atypical case of infection by Clostridium difficile
}

Maria Cristina Neri', Nadia Antoniotti', Maria Benvenuto' ${ }^{2}$ Sergio Frugoni ${ }^{3}$, Francesco Riboldi',Vito Noto'

I Azienda Servizi alla Persona Istituti Milanesi Martinitt e Stelline e Pio Albergo Trivulzio, IV U.O.C. - riabilitazione geriatrica, Milano

2 Azienda Servizi alla Persona Istituti Milanesi Martinitt e Stelline e Pio Albergo Trivulzio, I U.O.C. - riabilitazione cardiologica, Milano

3 Azienda Servizi alla Persona Istituti Milanesi Martinitt e Stelline e Pio Albergo Trivulzio, Servizio di Medicina di Laboratorio, Milano

Key Words: Clostridium difficile, caso atipico

Un caso atipico di infezione da Clostridium difficile

\section{SUMMARY}

Clostridium difficile is a Gram-positive human pathogenic bacterium and nosocomial pathogen; it is the causative agent diarrhoea, colitis and pseudo-membranous colitis associated with antibiotic therapy. The pathogenesis of diarrhoea linked to a Clostridium difficile infection is complex and only partly know. A 75 years old subject with biventricular defibrillator for atrial flutter-fibril slow dilated cardiomyopathy underwent, in february 2008, surgery of mitro-aortic replacement. The subject, in march 2009 , followed a rehabilitation therapy with aspirin and esomeprazole, as an outpatient, to Pio Albergo Trivulzio. The patient appeared lucid, oriented in time and space, in good general conditions; objectivity shows mild abdominal bloating, rectal examination negative; reported bowel function tend constipated with I-2 bowel movements per week with formed stools and recent episodes of melena. Blood tests showed anemia and positive research haemoglobin in stool. The patient underwent esophagogastroduodenoscopy and then rettosigmoidoscopia, and decided to carry out biopsies.

The sigmoid-rectal endoscopic picture was compatible with a diagnosis of "pseudo-membranous colitis hospitalization, On the basis of symptoms reported was required to search for toxins and bacterial culture for Clostridium difficile, resulting both positive. In literature are reported with increasing frequency of Clostridium difficile associated diarrhoea in patients home. The clinical case presented shows that in patients from home with symptoms vanished, the presence of formed stool does not exclude the possibility of infection by Clostridium difficile and is therefore useful and absolutely advisable to search for toxins and bacterial culture for Clostridium difficile.

\section{CASO CLINICO}

Il Clostridium difficile è noto come il principale agente responsabile di diarrea, colite e colite pseudomembranosa, associate a terapia antibiotica, soprattutto in ambiente ospedaliero.

La patogenesi della diarrea associata a Clostridium difficile, è complessa e solo in parte conosciuta: si suppone che in pazienti immunodepressi, la concomitante presenza di patologie disabilitanti e di terapia antibiotica, alteri la flora batterica del colon, creando condizioni favorevoli all'acquisizione o alla proliferazione del Clostridium difficile.

Diversi sarebbero i fattori di rischio, che possono includere, ad esempio, la severità della patologia concomitante, l'uso d'inibitori di pompa, la chirurgia gastrointestinale, l'età avanzata, l'ospedalizzazione prolungata, la degenza nei reparti di terapia intensiva, la presenza di sondino nasogastrico.

Nel marzo 2009 viene ricoverato nel reparto di riabilitazione geriatrica dell'Azienda Servizi alla Persona Pio Albergo Trivulzio, proveniente dal proprio domicilio, P.D., un uomo di 75 anni, sottoposto nel febbraio 2008 a sostituzione valvolare mitrale ed aortica e portatore di defibrillatore biventricolare per fibrillo-flutter atriale lento in miocardiopatia dilatativa. Al paziente, nel mese precedente al ricovero, vengono reimpiantate le valvole con bioprotesi per riscontro di endocardite con vegetazioni multiple e versamento pericardico, senza tamponamento.

$\mathrm{Al}$ ricovero, il paziente ha in corso terapia con esomeprazolo al dosaggio di 40mg/die ed aspirina al dosaggio di 100 $\mathrm{mg} / \mathrm{die}$; si presenta lucido, orientato nel tempo e nello spazio, in discrete condizioni generali; l'obiettività addominale evidenzia lieve meteorismo, l'esplorazione rettale è negativa; riferisce alvo tendenzialmente stitico con 1-2 evacuazioni alla settimana con feci formate e recente episodio di melena. Gli esami ematochimici mostrano anemizzazione e positività alla ricerca dell'emoglobina nelle feci.

Il paziente viene quindi sottoposto a esofagogastroduodenoscopia e rettosigmoidoscopia con riscontro di diverticolosi diffusa del sigma, multiple afte e lesioni pomfoidi biancogiallastre; in conseguenza di ciò, si decide per l'effettuazione di un prelievo bioptico (Figura I), durante il quale non si osservano sanguinamenti in atto.

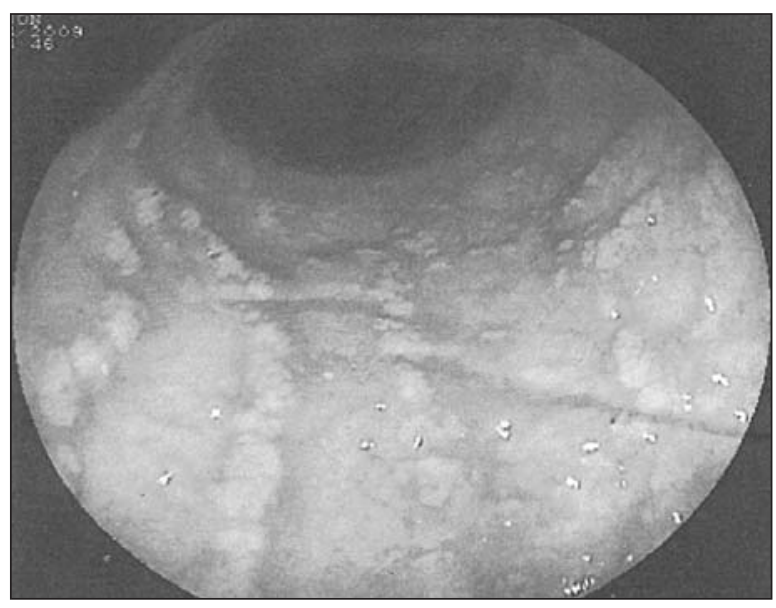

Figura I. Tratto del sigma con lesioni pomfoidi e multiple afte

Viene quindi effettuato un esame istologico, che riscontra la "Presenza di diffusi infiltrati linfoplasmocitari e granulocitari, con coesistenti fenomeni di aggressione granulocitaria dell'epitelio superficiale e ghiandolare".

Nei giorni successivi, per la comparsa di iperpiressia, viene introdotta una terapia antibiotica ex juvantibus con piperacillina/tazobactam e amikacina.

Sulla base della sintomatologia riferita, viene richiesta la ricerca delle tossine e l'esame colturale per $C$. difficile.

La ricerca delle tossine $\mathrm{A}$ e $\mathrm{B}$ è eseguita utilizzando il kit commerciale Toxins A \& B (Meridian) (3).

L'esame colturale è eseguito a temperatura ed in atmosfera idonea utilizzando piastre al sangue (CD Agar - bioMérieux). L’identificazione del microrganismo è eseguita utilizzando appropriate card del sistema automatico Vitek (bioMérieux). L'esame colturale per $C$. difficile e la ricerca nelle feci della tossina A e B risultano positive, mentre il quadro endoscopico retto-sigmoideo risulta compatibile con la diagnosi di "colite pseudo membranosa".

In considerazione dell'esame endoscopico, colturale ed istologico, si decide di proseguire la terapia antibiotica in atto, asso-

\section{Corresponding author: Sergio Frugoni}

Azienda Servizi alla Persona Istituti Milanesi Martinitt e Stelline e Pio Albergo Trivulzio, Servizio di Medicina di Laboratorio,

Via Trivulzio 15 - 20146 Milano - Tel.: +39-02-4029544 - Fax: +039- 02-4029549

E-mail: sergio.frugoni@pioalbergotrivulzio.it 
ciando ad essa una terapia orale con vancomicina al dosaggio di $250 \mathrm{mg} \mathrm{x} 4$ somministrazioni giornaliere, con il preciso scopo di trattare l'infezione da Clostridium difficile (1).

Al controllo endoscopico effettuato al termine della terapia, il quadro endoscopico e istologico risulta nella norma.

Anche in letteratura sono segnalati casi sempre più frequenti di diarrea associata a Clostridium difficile in pazienti domiciliari (2).

Lo spettro della patologia è molto vario: dal portatore asintomatico alla colite lieve o severa, fino alla colite pseudo membranosa.

In questi casi gli individui che acquisiscono il Clostridium difficile possono essere colonizzati, ovvero sviluppare la malattia: lo stato immunitario dell'ospite è un determinante importante per lo sviluppo della malattia.

Il caso clinico esposto dimostra come anche in pazienti provenienti dal domicilio con una sintomatologia sfumata, la presenza di feci formate non esclude la possibilità di infezione da $C$. difficile ed è quindi utile e assolutamente consigliabile effettuare la ricerca delle tossine e l'esame colturale per Clostridium difficile.

\section{BIBLIOGRAFIA}

1. Fekety R, Silva J, Kauffman C, Buggy B, Gunner Deery H. Treatment of antibiotic-associated Clostridium difficile colitis with oral vancomycin: comparison of two dosage regimens. Am Jour of Med. 1989; 86: 15-9.

2. Moro ML. Sorveglianza e controllo delle infezioni in Italia e in Europa. I.S.C. Atti. 2007; 51-5

3. Wilkins T, Lyerly D. Clostridium difficile testing: after 20years, still challenging. J Clin Microbiol, 2003; 41: 531-4. 